\title{
Harmonic balance Navier-Stokes aerodynamic analysis of horizontal axis wind turbines in yawed wind
}

\author{
Jernej Drofelnik ${ }^{1}$, Andrea Da Ronch ${ }^{1}$, M. Sergio Campobasso ${ }^{2}$ \\ ${ }^{1}$ University of Southampton, Faculty of Engineering and the Environment. Southampton SO17 1BJ, United Kingdom. ${ }^{2}$ University of \\ Lancaster, Department of Engineering. Lancaster LA1 4YW, United Kingdom.
}

\begin{abstract}
Multi-megawatt horizontal axis wind turbines often operate in yawed wind transients in which the resulting periodic loads acting on blades, drive-train, tower and foundation adversely impact on fatigue life. Accurately predicting yawed wind turbine aerodynamics and resulting structural loads can be challenging, and would require the use of computationally expensive high-fidelity unsteady Navier-Stokes Computational Fluid Dynamics. The high computational cost of this approach can be significantly reduced by using a frequency-domain framework. The paper summarizes the main features of the COSA Harmonic Balance Navier-Stokes solver for the analysis of open rotor periodic flows, presents initial validation results based on the analysis of the NREL Phase VI experiment, and it also provides a sample application to the analysis of a multi-megawatt turbine in yawed wind. The reported analyses indicate that the harmonic balance solver determines the considered periodic flows from 30 to 50 times faster than the conventional time-domain approach with negligible accuracy penalty to the latter. Copyright (c) 2017 John Wiley \& Sons, Ltd.
\end{abstract}

\section{KEYWORDS}

Harmonic Balance Navier-Stokes equations; horizontal axis wind turbine aerodynamics; yawed wind aerodynamics; NREL Phase VI wind turbine; NREL $5 \mathrm{MW}$ wind turbine

Correspondence

M. Sergio Campobasso, University of Lancaster, Department of Engineering. Lancaster LA1 4YW, United Kingdom.

E-mail: m.s.campobasso@lancaster.ac.uk

Received ...

\section{INTRODUCTION}

Wind energy is a key low-carbon energy source, playing a crucial role in lowering global greenhouse gas emissions, and it is now viewed as one of the most cost-effective climate change mitigation technologies. Current utility-scale horizontal axis wind turbines (HAWTs) already feature fairly high aerodynamic efficiencies; however, due to the spatial and temporal variability of the environmental conditions, HAWTs often experience unsteady flow conditions which induce fatigue and lower the energy harvest. Several of such regimes can be viewed as periodic, and typical examples include the blades rotating $a$ ) through stratifications of the atmosphere associated with the atmospheric boundary layer, with the wind velocity varying by as much as $6 \mathrm{~m} / \mathrm{s}$ over a $100 \mathrm{~m}$ rotor diameter [1], b) through the variable pressure field due to the downwind 
tower; $c$ ) in yawed wind, occurring when the freestream wind velocity is no longer orthogonal to the turbine rotor [2]. In all these cases, the fundamental frequency of the periodic excitation is a multiple of the rotor speed.

With regard to yawed wind, utility-scale HAWTs typically feature yaw control systems that monitor the wind direction, and turn the entire nacelle to re-align wind and rotor normal [2]. Yaw actuators, however, adjust the nacelle position after a relatively long time-interval from the yaw misalignment detection, and thus fatigue due to yaw misalignment can be significant. The yawed wind condition also reduces the power produced by the turbine and the reduction increases nonlinearly with the cosine of the yaw misalignment. Above certain wind speed-and turbine-dependent yaw misalignment thresholds, dynamic stall also occurs, and this aggravates further unsteady loads due to hysteretic force and moment cycles. Therefore, predicting with sufficient reliability yawed wind aerodynamics is paramount to wind turbine design. However, HAWT design methods largely rely on low-fidelity and semi-empirical models such as blade element momentum theory (BEMT) and dynamic stall models [3]. These techniques are extremely fast, but their reliance on the availability of high-quality airfoil data hinders their applicability to the design of radically new turbine configurations. Moreover, the predictions of low-fidelity methods are likely to be affected by significant uncertainty when dealing with complex threedimensional (3D) HAWT flows, such as yawed flows, featuring the so-called radial pumping effect [4]. Navier-Stokes (NS) Computational Fluid Dynamics (CFD) were shown to more reliably predict complex stalled HAWT flows in straight wind [5].

Most published NS CFD investigations of yawed HAWT flows focused on stall-regulated turbines, most notably the NREL Phase VI [6] and MEXICO [7] wind tunnel experiments. The measured flow data of these experiments provide valuable insight into unsteady HAWT aerodynamics and also invaluable information for validating new codes used in wind turbine analysis and design. For example, Madsen et al. [8] presented the yawed flow analyses of an outdoor 100 $\mathrm{kW}$ wind turbine and the NREL Phase VI wind turbine. CFD NS results, experimental data and results obtained with lowfidelity codes, including a BEMT code, were compared. The study highlighted that NS CFD results agreed significantly better with measured data than low-fidelity results. Le Pape and Gleize [9] performed NS CFD yawed flow analyses of the NREL Phase VI wind turbine. The experimental data were in fairly good agreement with the CFD simulations. Sezer-Uzol et al. [10] performed inviscid and a large eddy simulation yawed wind flow analyses of the NREL Phase VI wind turbine, obtaining a good agreement between measurements and simulations for the sectional pressure coefficient distributions. Tsalicoglou et al. [11] conducted yawed flow CFD analyses of the MEXICO wind turbine, and reported a good agreement between their NS CFD and experimental data. Yu et al. [12] studied the yawed rotor flow of the NREL Phase VI turbine using overset grid NS CFD simulations and a zonal laminar-to-turbulent transition model. They highlighted that blade loading is significantly reduced in yawed conditions, and obtained an overall good agreement of computed and measured data for all considered wind speeds and yaw angles. The MEXNEXT-II report [13] includes several studies of yawed wind flow for the NREL Phase VI, Mexico and the DTU $100 \mathrm{~kW}$ Tellus turbines, and recommends that CFD methods should be adopted for the analysis of stalled and yawed operating conditions, due to their better prediction of the blade aerodynamic forces.

The drawback of using NS CFD for analyzing yawed flows is its high computational cost. Time-domain (TD) NS simulation of HAWT periodic flows require long runtimes as several rotor revolutions need to be simulated before a periodic state is achieved. This runtime can be significantly reduced by solving the governing equations in the frequency-domain. A widespread method of this type is the harmonic balance $(H B)$ NS technology, initially introduced for turbomachinery blade aeroelasticity [14] and successively also used for multi-stage turbomachinery aerodynamics $[15,16]$, several vibratory motion modes of aircraft configurations [17, 18, 19], and recently also for wind turbine aerodynamics [20,21] and aeroelasticity [22]. The use of the $H B$ NS method for the simulation of this type of periodic flows has been shown to reduce by one to two orders of magnitude CFD runtimes with respect to conventional $T D$ NS analyses. Several other nonlinear frequency-domain NS methods exist and have been applied in the abovesaid areas, as more extensively reported in [20].

The use of the $H B$ method for reducing the runtime of NS simulations of the yaw-induced periodic flow past HAWT rotor blade sections was first investigated in [20]. The study used the two-dimensional (2D) compressible laminar NS 
equations with low-speed preconditioning, and reported a tenfold speed-up of the periodic flow calculation achieved by using the $H B$ rather than the $T D$ method. Howison and Ekici [21] used the Spalart-Allmaras turbulence model and lowspeed preconditioning, and, later, the Spalart-Allmaras model and a zonal transition model [23] to successfully analyze the 2D periodic flow past a pitching S809 airfoil with the $H B$ approach, whereas Menter's shear stress transport (SST) turbulence model was used in [24] for the 2D HB NS analyses of an inboard section of a utility-scale HAWT blade in yawed wind. A 3D HB NS approach was used in [22] to assess the flutter characteristics of the 1.5 MW WindPACT rotor blade. These studies highlight a growing interest in the use of this high-fidelity approach for the analysis of HAWT periodic aerodynamics. The main objectives and novel features of this paper are to $a$ ) assess the robustness and the solution reliability of the high-fidelity and computationally cost-effective 3D HB NS technology applied to the prediction of the periodic rotor loads due to realistic turbulent yawed wind, and $b$ ) quantifying the acceleration of the $H B$ over the $T D$ analysis achievable by using settings of the $H B$ analysis yielding prediction accuracy comparable with that of its $T D$ counterpart.

The $T D$ and $H B$ integral form of the Reynolds-averaged Navier-Stokes (RANS) equations and the SST turbulence model in a Cartesian rotating frame are presented in Section 2, and a brief description of the numerical method is provided in Section 3. Section 4 defines the boundary conditions used by the CFD code, with particular emphasis on the far field and multi-frequency periodicity boundary conditions. Section 5 presents a validation study of the CFD code based on the comparison of CFD results and measured data of an upwind rotor configuration of the NREL Phase VI turbine. Measured data and steady CFD predictions are compared for two zero-yaw set-ups, whereas measurements and TD simulations are compared for one yawed wind regime. The accuracy and computational benefits of the developed $H B$ RANS technology are assessed in Section 6 by using the $H B$ solver to analyze the NREL Phase VI yawed flow of Section 5. A yawed flow regime of the NREL 5 MW turbine [25] is also analyzed to provide a sample demonstration of this CFD method for utility-scale machines. A summary of the study and future perspectives are provided in the closing section.

\section{GOVERNING EQUATIONS}

\subsection{Time-domain equations}

The 3D compressible NS equations are a system of conservation laws expressing the conservation of mass, momentum and energy in a viscous fluid flow. Averaging the NS equations on the turbulence time-scales, yields the RANS equations, which are formally similar to the NS equations but feature an additional term, the Reynolds stress tensor accounting in a mean fashion for the effects of turbulence. Making use of Boussinesq's approximation, this tensor is expressed as the product of an eddy viscosity and the strain rate tensor based on the mean velocity field. In the COSA CFD code used in this study, the former variable is computed with the two-equation $k-\omega$ SST turbulence model. Thus, turbulent compressible flows are determined by solving a system of $N_{p d e}=7$ partial differential equations (PDEs) and an equation of state linking fluid density, pressure and internal energy. Although compressibility effects in HAWT flows may presently be relatively small, due to the blade tip speed of modern HAWTs not exceeding the Mach 0.3 threshold, the compressible flow formulation was adopted in COSA to develop and maintain a single code for both low-speed [26] and high-speed [17] problems.

In many applications involving rotational body motion (e.g. turbomachinery, helicopter and HAWT rotor flows), it is convenient to formulate the governing equations in a rotating frame of reference. Using this approach, the grid position is fixed during the analysis. When formulating the governing equations in a rotating frame, one can express the relative flow velocity vector either in the relative or the absolute frame [27]. The two formulations are mathematically equivalent, but representing the relative fluid velocity in the absolute frame is numerically more convenient for open rotor applications. Therefore, the relative velocity vector is expressed in an absolute frame in this study. 
The Arbitrary Lagrangian-Eulerian (ALE) integral form of the system of the time-dependent RANS and SST equations is written in a rotating Cartesian coordinate system, rotating about an axis coinciding with its $z$ axis with constant angular velocity $\underline{\Omega}$, as depicted in Fig. 1 .

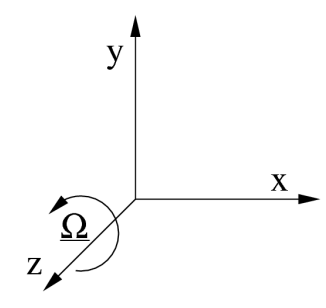

Figure 1. Rotating Cartesian coordinate system.

Given a control volume $C$ with boundary $S$ in the considered rotating frame, the ALE integral form of the system of time-dependent RANS and SST equations is:

$$
\frac{\partial}{\partial t}\left(\int_{C} \mathbf{U} d C\right)+\oint_{S}\left(\underline{\mathbf{\Phi}}_{c}-\underline{\mathbf{\Phi}}_{d}\right) \cdot d \underline{S}-\int_{C} \mathbf{S}_{r} d C=0
$$

where $\mathbf{U}=\left[\begin{array}{lllll}\rho & \rho \underline{\mathrm{u}}^{T} & \rho E & \rho k & \rho \omega\end{array}\right]^{T}$ is the array of conservative variables, the superscript $T$ denotes the transpose operator, and the symbols $\rho, \underline{u}, E, k$ and $\omega$ denote respectively density, absolute velocity vector, and total energy, turbulent kinetic energy and specific dissipation rate of turbulent kinetic energy per unit mass. The total energy is $E=e+(\underline{\mathrm{u}} \cdot \underline{\mathrm{u}}) / 2+k$, where $e$ denotes the internal energy per unit mass; the perfect gas law is used to express the static pressure $p$ as a function of $\rho, E, k$ and the mean flow kinetic energy per unit mass $(\underline{u} \cdot \underline{u}) / 2$ [28]. The generalized convective flux vector $\underline{\Phi}_{c}$ is:

$$
\underline{\boldsymbol{\Phi}}_{c}=\left[\begin{array}{c}
\rho\left(\underline{\mathrm{u}}-\underline{\mathrm{u}}_{b}\right)^{T} \\
\rho\left(\underline{\mathrm{u}}-\underline{\mathrm{u}}_{b}\right)^{T} \underline{\mathrm{u}}+p I \\
\rho E\left(\underline{\mathrm{u}}-\underline{\mathrm{u}}_{b}\right)^{T}+p \underline{\mathrm{u}}^{T} \\
\rho k\left(\underline{\mathrm{u}}-\underline{\mathrm{u}}_{b}\right)^{T} \\
\rho \omega\left(\underline{\mathrm{u}}-\underline{\mathrm{u}}_{b}\right)^{T}
\end{array}\right]
$$

where $I$ is the $(3 \times 3)$ identity matrix. The boundary velocity $\underline{u}_{b}$ is given by:

$$
\underline{\mathrm{u}}_{b}=\underline{\Omega} \times \underline{\mathrm{r}}
$$

in which $\underline{r}$ denotes the position vector in the rotating frame. The definition of generalized diffusive flux vector $\underline{\boldsymbol{\Phi}}_{d}$ is reported in [24], and the source term $\mathbf{S}$ is given by:

$$
\mathbf{S}=\left[\begin{array}{lllllll}
0 & -\rho \Omega v & \rho \Omega u & 0 & 0 & S_{k} & S_{\omega}
\end{array}\right]^{T}
$$

where $\Omega$ denotes the modulus of $\underline{\Omega}$, and $S_{k}$ and $S_{\omega}$ denote respectively the source terms of the $k$ and $\omega$ equations of the SST turbulence model. The definitions of $S_{k}$ and $S_{\omega}$ are provided in [26].

In COSA the molecular viscosity is computed with Sutherland's law using the local static temperature. Despite the fairly small variations of static temperature and molecular viscosity in the CFD analyses reported below, the viscosity was allowed to vary to maintain a simple and general structure of the code, and also due to the fairly small computational savings associated with using a constant viscosity. 


\subsection{Harmonic balance equations}

The detailed derivation of the $H B$ RANS and SST equations in a rotating frame follows the same steps of that in the inertial frame, which was provided in numerous previous studies, including [14] and [20]. Here only a qualitative description of the derivation is provided.

The sought periodic flow field is written as a truncated Fourier series with $\left(2 N_{H}+1\right)$ spatial position-dependent components, namely $N_{H}$ sinusoidal components, $N_{H}$ cosinusoidal components, and a time-independent term. The parameter $N_{H}$ is user-given, and the fundamental frequency $\Omega$ of the Fourier series is the known frequency of the considered harmonic excitation. The Fourier series approximation of the solution is inserted in the $T D$ governing equations expressed by System (1). This operation results in the original system of $N_{p d e}$ time-dependent PDEs becoming a system of $\left[N_{p d e} \times\left(2 N_{H}+1\right)\right]$ time-independent PDEs, the solution of which yields the $\left(2 N_{H}+1\right)$ components of the truncated Fourier series. However, writing the $H B$ RANS and turbulence model equations in the Fourier space turns out to be challenging, due to the high level of nonlinearity of the equations involved; for this reason, [14] proposed to re-cast the $H B$ CFD equations in the time-domain. Indeed, this choice simplifies substantially the construction of the $H B$ equations and also the implementation of this technology in an existing CFD code. Formally, re-casting the $H B$ equations in the time-domain, results in the $H B$ equations becoming a system of $\left(2 N_{H}+1\right)$ steady flow problems, and the $H B$ solution becoming a set of equally spaced flow states or snapshots of the sought periodic flow. Once determined, the $H B$ solution can be re-cast in the Fourier space by using a suitably defined Fourier transform.

Applying the aforementioned procedure, one finds that the desired $H B$ form of the considered conservation laws is:

$$
\Omega D\left(\int_{C_{H}} \mathbf{U}_{H} d C_{H}\right)+\oint_{S_{H}}\left(\underline{\mathbf{\Phi}}_{c H}-\underline{\mathbf{\Phi}}_{d H}\right) \cdot d \underline{S}_{H}-\int_{C_{H}} \mathbf{S}_{H} d C_{H}=0
$$

The unknown array $\mathbf{U}_{H}$ is made up of $2 N_{H}+1$ periodic flow snapshots at $2 N_{H}+1$ equally spaced times $t_{n}$ given by:

$$
t_{n}=\frac{n}{\left(2 N_{H}+1\right)} \frac{2 \pi}{\Omega}, \quad n=0,1, \ldots, 2 N_{H}
$$

The structure of the array $\mathbf{U}_{H}$ is thus: $\mathbf{U}_{H}=\left[\tilde{\mathbf{U}}\left(t_{0}\right)^{T} \tilde{\mathbf{U}}\left(t_{1}\right)^{T} \ldots \tilde{\mathbf{U}}\left(t_{N_{H}}\right)^{T}\right]^{T}$, and is the same of that of all other variables with a subscript $H$ appearing in Eq. (5). The spectral operator $D$ is a $\left[\left(2 N_{H}+1\right) \times\left(2 N_{H}+1\right)\right]$ antisymmetric matrix, defined in [20], which couples all $\left(2 N_{H}+1\right)$ flow snapshots. It can be shown that the entry $(m, n)$ of $D$ is:

$$
D_{m n}=\frac{2}{2 N_{H}+1} \sum_{k=1}^{N_{H}} k \sin \left(\frac{2 \pi k(n-m)}{2 N_{H}+1}\right), \quad m, n=0,2 N_{H}
$$

Moving from the conventional $T D$ to the $H B$ formulation of the governing equations, one has to solve $\left[N_{p d e} \times\right.$ $\left.\left(2 N_{H}+1\right)\right]$ time-independent or steady PDEs rather than $N_{\text {pde }}$ time-dependent PDEs. The solution of each steady PDE requires substantially less computational work than that of a time-dependent PDE. This cost reduction outweighs the burden of solving more PDEs in the $H B$ case and thus the overall computational cost of solving the $H B$ equations turns out to be smaller than that required for solving the $T D$ equations. Therefore, turbulent periodic flows can be computed significantly faster using the $H B$ rather than the $T D$ approach in many engineering applications. This is because of the avoidance of the physical transient leading to the periodic state, and the possibility of using multi-frequency periodicity boundary conditions enabling the size reduction of the computational domain, as shown below.

\section{NUMERICAL SOLUTION}

The finite volume cell-centered parallel NS CFD COSA code solves both the TD RANS and SST equations [28, 29, 30] and their $H B$ counterparts [26] using structured multi-block grids. The discretization of the convective fluxes of both 
RANS and SST PDEs uses Van Leer's MUSCL extrapolations and Roe's flux-difference splitting with Van Albada's flux limiter. The discretization of the diffusive fluxes and the turbulent source terms uses central finite-differencing [28].

The integration of the steady and $H B$ RANS and SST equations is performed in a fully-coupled fashion using explicit Runge-Kutta time-marching, with local time-stepping, implicit residual smoothing and multigrid for convergence acceleration. $T D$ problems are solved using Jameson's second-order dual-time stepping.

In rotor flow analyses, COSA computes the cell face velocities $\underline{u}_{b}$ in Eq. (2) using the freestream-capturing geometric relations of [31] to ensure global conservation for time-dependent flows. Aditionally, both the source terms depending on $\Omega$ in Eq. (4) and the cell face velocities are nonzero also for steady rotor flows.

\section{BOUNDARY CONDITIONS}

\subsection{Wall boundary condition}

At viscous wall boundaries such as those associated with blade surfaces, the no-slip condition requires that fluid and boundary velocities be equal. Therefore, for steady, $T D$, and $H B$ problems the local fluid velocity is computed with Eq. (3). At wall cell faces, pressure is determined with second order extrapolations, temperature is determined by imposing a zero heat flux condition, and density is computed with the perfect gas equation of state. The turbulent kinetic energy at the wall is set to zero and the specific dissipation rate $\omega$ is computed as proposed in [32]. Neither wall nor any damping functions are used, and the RANS and turbulence model equations are integrated all the way down to wall boundaries. The minimum wall distances of all grids used in the CFD analyses reported below are such that the nondimensional minimum wall distance $y^{+}$is less than 1 around all wall boundaries. This ensures that the laminar sublayer, which is the innermost layer of turbulent boundary layers, is well resolved in all analyses. In $H B$ analyses, the wall boundary condition (BC) is applied to each of the $2 N_{H}+1$ flow snapshots and, due to the use of a relative frame formulation, the flow velocity at solid boundaries is the same at all times $t_{n}$ defined by Eq. (6). The flow velocity at solid boundaries is also the same at all times of $T D$ analyses, as these analyses are also based on a relative frame formulation.

\subsection{Freestream boundary condition}

The implementation of the freestream BC is based on one-dimensional Riemann invariants and follows closely the model of [33]. A additional key feature of the present study study, however, is the definition of the components of the freestream velocity for the $T D$ and $H B$ solvers. Solving the governing equations in a rotating frame implies that in the case of yawed flow the freestream velocity in the relative frame varies with the azimuthal position of the rotor. Let $u_{\infty}^{A}, v_{\infty}^{A}$, and $w_{\infty}^{A}$ denote respectively the (constant) $x, y$ and $z$ components of the freestream velocity in an absolute Cartesian frame. The velocity components $u_{\infty}, v_{\infty}$, and $w_{\infty}$ to be used as input for the freestream $\mathrm{BC}$ in the rotating frame are:

$$
\left[\begin{array}{l}
u_{\infty} \\
v_{\infty} \\
w_{\infty}
\end{array}\right]=\left[\begin{array}{ccc}
\cos \theta(t) & -\sin \theta(t) & 0 \\
\sin \theta(t) & \cos \theta(t) & 0 \\
0 & 0 & 1
\end{array}\right]\left[\begin{array}{c}
u_{\infty}^{A} \\
v_{\infty}^{A} \\
w_{\infty}^{A}
\end{array}\right]
$$

where $\theta(t)=\Omega t$. It is noted that the components $u_{\infty}, v_{\infty}$, and $w_{\infty}$ define the time-dependent freestream velocity vector in the relative frame using an absolute frame representation, and that the variability of this vector is the source of unsteadiness in $T D$ and $H B$ yawed wind simulations. For $H B$ analyses, the time variable $t$ takes the $2 N_{H}+1$ values defined by Eq. (6), and the freestream velocity components obtained from Eq. (8) for $t=t_{n}$ are used to build the freestream boundary data for the $n^{t h}$ flow snapshot. The steady problem is retrieved setting $u_{\infty}^{A}=v_{\infty}^{A}=0$. This yields $w_{\infty}=w_{\infty}^{A}$ and corresponds to zero yaw misalignment. 


\subsection{Periodicity boundary condition}

In the case of steady rotor flow problems, the circumferential flow periodicity enables one to reduce the size of the computational domain by a factor proportional to the number of of rotor blades $N_{b}$, as the use of a periodicity BC enables the use of a single grid sector to simulate the flow past a single blade. The sector features two periodic boundaries, and periodicity is achieved by enforcing that the flow conditions at both boundaries be the same. Since COSA is a cellcentered code and presently uses periodic grids (i.e. the grid nodes of one periodic boundary coincide with those on the other periodic boundary performing a rotation of $2 \pi / N_{b}$ radians), the steady periodicity BC is implemented by copying the scalar flow variables of the two grid cell interior layers adjacent to each periodic boundary to the corresponding two layers of auxiliary (i.e. fictitious) cells of the other periodic boundary. A rigid body rotation, however, has to be applied to the flow velocity vector. Denoting by $\underline{u}_{p 1}$ the velocity vector at the center of one interior cell adjacent to periodic boundary 1 and $\underline{u}_{p 2}$ that at the center of the corresponding auxiliary cell adjacent to periodic boundary 2 , one has:

$$
\underline{\mathrm{u}}_{p 2}=\tilde{R} \underline{\mathrm{u}}_{p 1}
$$

with

$$
\tilde{R}=\left[\begin{array}{ccc}
\cos \theta_{s} & \sin \theta_{s} & 0 \\
-\sin \theta_{s} & \cos \theta_{s} & 0 \\
0 & 0 & 1
\end{array}\right]
$$

and

$$
\theta_{s}=\frac{2 \pi}{N_{b}}
$$

In the case of unsteady periodic rotor flows, the flow field on the two periodic boundaries at a given time is different and the steady periodicity condition discussed above cannot be used. Thus, using the $T D$ approach, one must simulate the flow field of the entire rotor. With the frequency-domain $H B$ approach, however, the steady periodicity BC can be generalized to a multi-frequency periodicity BC (MFPBC), which enables the use of a single rotor sector in the simulation and was previously applied to the $H B$ NS analysis of helicopter rotor flows [34]. The MFPBC relies on the fact that for each harmonic retained in the truncated Fourier reconstruction of the sought periodic flow there exists a constant phase between the flow field at the two periodic boundaries. This can be illustrated by considering the rotation of a three-blade HAWT rotor in yawed wind in the absolute frame depicted schematically in Fig. 2, where the left plot shows the rotor at time $t$ and the right plot at time $t+\Delta t_{s}$, with the time-interval $\Delta t_{s}$ given by

$$
\Delta t_{s}=\frac{T}{N_{b}}
$$

and the rotor revolution period $T$ given by $T=2 \pi / \Omega$. Labels $\theta_{p 1}$ and $\theta_{p 2}$ denote the angular positions of the periodic boundaries of the grid sector of blade B1 in the rotating frame.

Inspections of the two plots of Fig. 2 clearly indicates that:

$$
\mathbf{Q}\left(\theta_{p 2}, t\right)=\mathbf{Q}\left(\theta_{p 1}+\theta_{s}, t\right)=\mathbf{Q}\left(\theta_{p 1}, t+\Delta t_{s}\right)
$$

where $\mathbf{u}$ is the array of discrete flow variables at a periodic boundary and Eq. (13) holds for given values of the $z$ coordinate and normal distance from the $z$ axis. In the $H B$ framework [20], the array $\mathbf{u}(\theta, t)$ is given by the truncated Fourier series:

$$
\mathbf{Q}(\theta, t)=\hat{\mathbf{Q}}_{0}(\theta)+\sum_{n=1}^{N_{H}}\left(\hat{\mathbf{Q}}_{2 n-1}(\theta) \cos (n \Omega t)+\hat{\mathbf{Q}}_{2 n}(\theta) \sin (n \Omega t)\right)
$$




\section{Rotor at time $\mathbf{t}$}

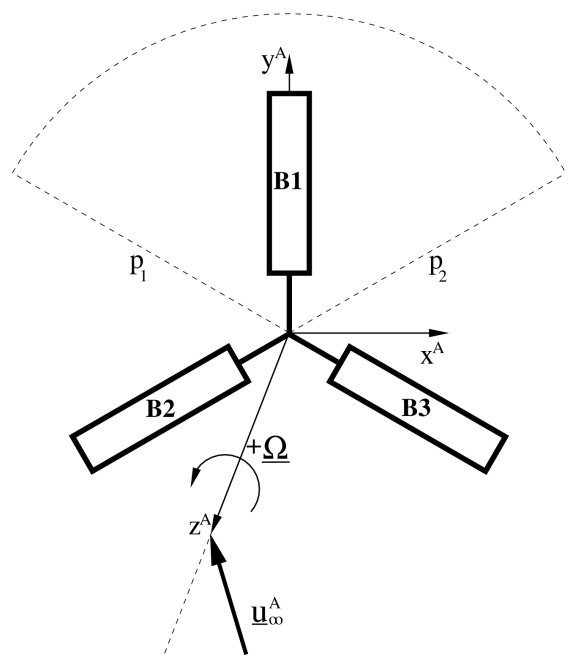

Rotor at time $t+\Delta t$

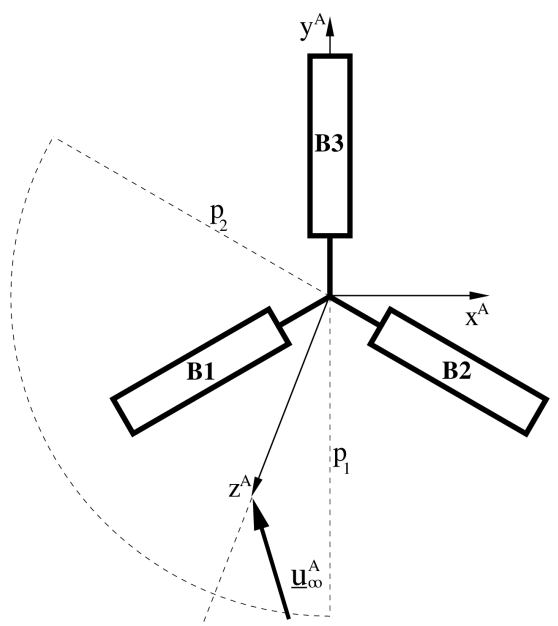

Figure 2. Rotor position at time $t$ (left) and time $t+\Delta t_{s}$ (right).

where $\hat{\mathbf{Q}}_{0}$ is the mean value of the unknown periodic solution, and $\hat{\mathbf{Q}}_{2 n-1}$ and $\hat{\mathbf{Q}}_{2 n}$ are respectively real and imaginary parts of the $N_{H}$ complex harmonics expressing its time variations. Inserting Eq. (14) into Eq. (13) and matching terms with the same frequency yields the equations of the MFPBC, namely:

$$
\begin{aligned}
\hat{\mathbf{Q}}_{0}\left(\theta_{p 2}\right) & =\hat{\mathbf{Q}}_{0}\left(\theta_{p 1}+\theta_{s}\right)=\hat{\mathbf{Q}}_{0}\left(\theta_{p 1}\right) \\
\hat{\mathbf{Q}}_{2 n-1}\left(\theta_{p 2}\right) & =\hat{\mathbf{Q}}_{2 n-1}\left(\theta_{p 1}+\theta_{s}\right)=\hat{\mathbf{Q}}_{2 n-1}\left(\theta_{p 1}\right) \cos \left(n \theta_{s}\right)+\hat{\mathbf{Q}}_{2 n}\left(\theta_{p 1}\right) \sin \left(n \theta_{s}\right) \\
\hat{\mathbf{Q}}_{2 n}\left(\theta_{p 2}\right) & =\hat{\mathbf{Q}}_{2 n}\left(\theta_{p 1}+\theta_{s}\right)=-\hat{\mathbf{Q}}_{2 n-1}\left(\theta_{p 1}\right) \sin \left(n \theta_{s}\right)+\hat{\mathbf{Q}}_{2 n}\left(\theta_{p 1}\right) \cos \left(n \theta_{s}\right)
\end{aligned}
$$

Equations (14) highlight that the MFPBC is applied in the frequency-domain and consists of applying a phase shift $n \theta_{s}$ to the amplitude of the $n^{t h}$ complex harmonic when copying it from periodic boundary 1 to periodic boundary 2 . Moreover, since the $H B$ code solves for the $2 N_{H}+1$ snapshots of the periodic flow field in the time-domain, one must apply a Fourier transform $F_{H}^{-1}$ to obtain the complex amplitudes $\tilde{\mathbf{Q}}$ from the snapshots $\hat{\mathbf{Q}}$ before applying the MFPBC, and the inverse transform $F_{H}$ to move back to the time-domain after applying the MFPBC. The expressions of $F_{H}^{-1}$ and $F_{H}^{-1}$ are provided in [20]. It is also noted that the geometric rotation given by Eq. (9) also needs to be applied to each complex harmonic amplitude of the flow velocity before reverting back to the $T D$ representation of the solution.

\section{VALIDATION OF TIME-DOMAIN SOLVER}

Two zero-yaw regimes and one yawed regime of one rotor configuration of the NREL Phase VI wind turbine experiments [6] are examined herein for validating the COSA steady and TD solvers. The Phase VI rotor has radius $R=5.029 \mathrm{~m}$, and features two blades with non-linear twist and linear taper distributions. The considered upwind rotor set-up has blades mounted with a tip pitch of $3^{\circ}$ towards feather, and rotor cone angle of $0^{\circ}$. This stall-regulated turbine has rated power of $19.8 \mathrm{~kW}$ and cut-in wind speed $v_{\infty}$ of $6 \mathrm{~m} / \mathrm{s}$. The operating conditions of the two steady regimes are reported in Table I. Here $\left|\underline{u}_{\infty}^{A}\right|$ is the magnitude of the freestream velocity, $\mu$ is the dynamic viscosity, and $R e$ is the Reynolds number based on the tip chord, and the relative flow velocity at the tip. The considered yawed regime is that of $\left|\underline{u}_{\infty}^{A}\right|=7 \mathrm{~m} / \mathrm{s}$ with a yaw misalignment of $30^{\circ}$. 
Table I. NREL Phase VI HAWT: examined operating conditions.

\begin{tabular}{|c|c|c|c|c|}
\hline$\left|\underline{\mathrm{u}}_{\infty}^{A}\right|(\mathrm{m} / \mathrm{s})$ & $\Omega(\mathrm{RPM})$ & $\rho\left(\mathrm{kg} / \mathrm{m}^{3}\right)$ & $\mu(\mathrm{kg} / \mathrm{ms})$ & $\mathrm{Re}$ \\
\hline 7 & 71.9 & 1.246 & $1.769 \times 10^{-5}$ & $0.96 \times 10^{6}$ \\
\hline 13 & 72.1 & 1.227 & $1.781 \times 10^{-5}$ & $0.98 \times 10^{6}$ \\
\hline
\end{tabular}

In the CFD simulations analyzed below, only the rotor was modeled and zero wind shear was assumed. Figure 3 reports views of the blade geometry and sector grid used for the steady zero-yaw simulations, and the $H B$ yawed wind analyses discussed in Section 6. The grid has 17,006, 592 cells. Each blade airfoil is discretized with 256 mesh intervals and the distance $d_{w}$ of the first grid points off the airfoil from the airfoil itself is about $1 \cdot 10^{-5} c$, with $c$ being the local airfoil chord. This choice guarantees a minimum nondimensional wall distance $y^{+}$less than 1 on the entire blade surface. In the spanwise direction, the grid has 128 intervals on the blade surface and 96 intervals from the tip to the cylindrical far field boundary. The blade tip was modeled using a sharp cut. A coarse $2.1 \mathrm{M}$-cell grid was also obtained from the $17 \mathrm{M}$-cell fine grid by removing every second line in all three directions. Further detail on these coarse and fine grids are available in [35]. The yawed wind $T D$ analysis used a $34 \mathrm{M}$-cell full rotor fine grid obtained by adding to the $17 \mathrm{M}$-cell sector grid described above the same sector grid rotated by $180^{\circ}$.

The dimensions of the physical domain and the BCs applied to its boundaries are reported in Fig. 3. These domain dimensions were selected on the basis of the outcome of a sensitivity study aiming at finding the minimum far field distances yielding negligible spurious reflections from such boundaries. This was accomplished starting from a relatively small distance of the farfield boundaries from the blade, and increasing such distance until the computed flow field past the blade became independent of the domain size. The top left plot of Fig. 3 shows that the hub was modeled as a zerothickness axially-short cylindrical surface, and an inviscid wall BC was used on its inner and outer sides.

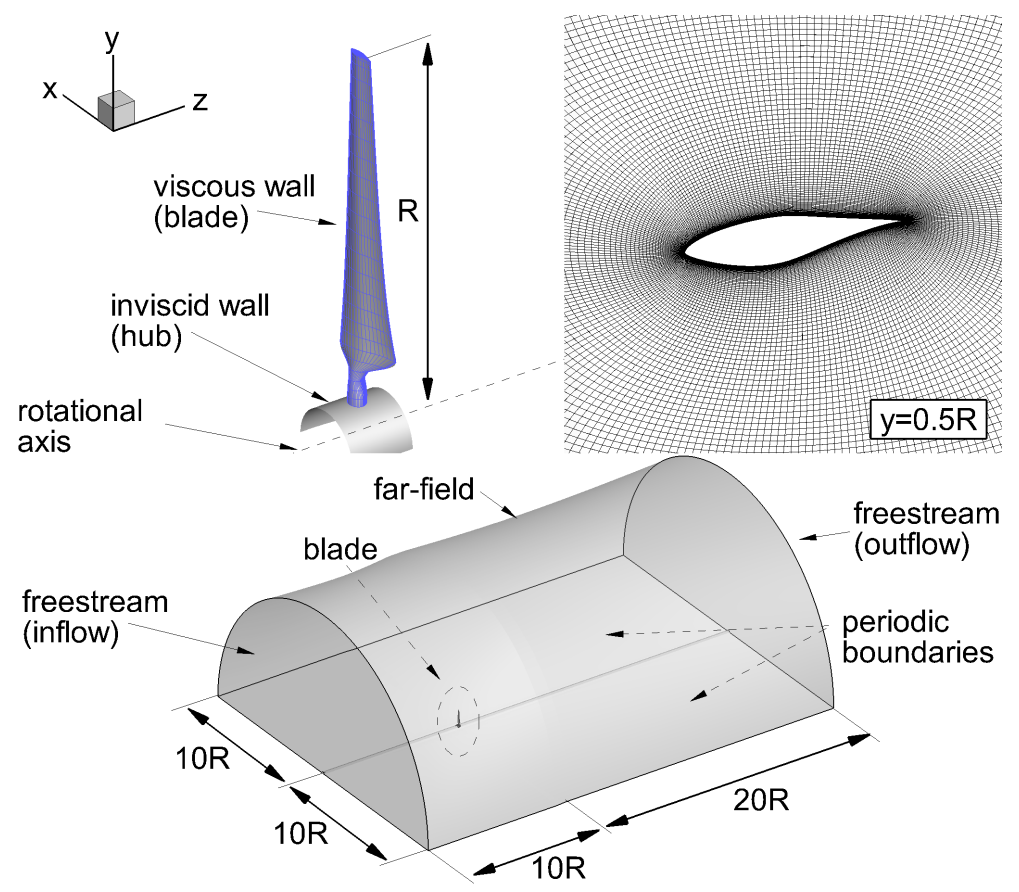

Figure 3. NREL Phase VI HAWT: blade geometry (top left), airfoil grid at $50 \%$ tip radius (top right), and domain dimensions and boundary conditions (bottom). 
The contribution $F_{z}$ of one blade to the rotor thrust and the contribution $M_{z}$ to the rotor torque computed with the zeroyaw steady simulations for the two considered freestream velocities are reported in Tab. II, which compares the coarse and fine grid estimates with measured data. It is noted that coarse and fine grid results for the freestream speed of $7 \mathrm{~m} / \mathrm{s}$ differ by less than $3 \%$, whereas larger differences occur at $13 \mathrm{~m} / \mathrm{s}$, where the two numerical estimates of the torque differ by about $10 \%$. Both coarse and fine grid estimates are close to the measured data within the known uncertainty of these measurements (at $7 \mathrm{~m} / \mathrm{s}$ the uncertainty affecting both $F_{z}$ and $M_{z}$ is about $2.5 \%$ of the mean values indicated in Tab. II; at $13 \mathrm{~m} / \mathrm{s}$ the uncertainty levels affecting $F_{z}$ and $M_{z}$ are about $15 \%$ and $27 \%$, respectively, of the mean values indicated in Tab. II). The better agreement of coarse and fine grid estimates for the freestream speed of $7 \mathrm{~m} / \mathrm{s}$ occurs because at this regime the flow is predominantly attached, whereas the significant amount of stall at $13 \mathrm{~m} / \mathrm{s}$ requires a finer grid resolution.

Table II. NREL Phase VI HAWT: measured and steady CFD thrust $F_{z}$ and torque $M_{z}$ of one blade.

\begin{tabular}{|c|c|c|c|c|c|c|}
\hline \multirow{2}{*}{$v_{\infty}(m / s)$} & \multicolumn{2}{|c|}{ COSA coarse } & \multicolumn{2}{c|}{ COSA fine } & \multicolumn{2}{c|}{ experiment } \\
\cline { 2 - 7 } & $F_{z}(k N)$ & $M_{z}(k N m)$ & $F_{z}(k N)$ & $M_{z}(k N m)$ & $F_{z}(k N)$ & $M_{z}(k N m)$ \\
\hline 7 & 555.8 & 354.2 & 544.6 & 341.2 & 578.5 & 402.0 \\
\hline 13 & 966.1 & 660.3 & 999.0 & 730.0 & 1012.2 & 679.3 \\
\hline
\end{tabular}

Computed and measured spanwise profiles of normal force coefficient $C_{N}$ and tangential force coefficient $C_{T}$ for $\left|\underline{\mathrm{u}}_{\infty}^{A}\right|=7 \mathrm{~m} / \mathrm{s}$ are examined respectively in the top left and top right plots of Fig. 4, whereas the profiles of the same variables to $\left|\underline{u}_{\infty}^{A}\right|=13 \mathrm{~m} / \mathrm{s}$ are examined in the two bottom plots of the same figure. For both computed and measured data, the definitions of $C_{N}$ and $C_{T}$ are respectively

$$
\begin{aligned}
C_{N} & =\sum_{i=1}^{n_{s}}\left(\frac{c_{p_{i}}+c_{p_{i+1}}}{2}\right)\left(x_{i+1}^{\prime}-x_{i}^{\prime}\right) \\
C_{T} & =\sum_{i=1}^{n_{s}}\left(\frac{c_{p_{i}}+c_{p_{i+1}}}{2}\right)\left(y_{i+1}^{\prime}-y_{i}^{\prime}\right)
\end{aligned}
$$

where $x^{\prime}$ and $y^{\prime}$ denote respectively the coordinate along the airfoil chord and that normal to the airfoil chord, the subscript $i$ refers to the midpoint of a mesh interval on the airfoil in the numerical estimate and the position of a pressure tap in the experimental estimate, and $n_{s}$ indicates the number of mesh intervals along the airfoil in the numerical estimate and the number of pressure taps in the experimental estimate. The definition of the pressure coefficient $c_{p}$ is:

$$
c_{p}=\frac{p^{a}-p_{\infty}}{\frac{1}{2} \rho_{\infty}\left[\left|\underline{\mathbf{u}}_{\infty}^{A}\right|^{2}+(\Omega r)^{2}\right]}
$$

where $p^{a}$ and $p_{\infty}$ denote respectively airfoil and freestream static pressure, and $r$ denotes the radial position along the blade. Figure 4 shows that the agreement of computed estimates of $C_{N}$ and the measured values of the same variable is excellent for both freestream wind speeds, whereas the fine and coarse grid estimates of $C_{T}$ present some more significant differences, with the fine grid estimate being marginally closer to measured data. The differences between the coarse and fine grid predictions of the tangential force are comparable, but those at $7 \mathrm{~m} / \mathrm{s}$ occur at the inboard part of the blade that produces relatively low torques, whereas those at $13 \mathrm{~m} / \mathrm{s}$ occur at the outboard part of the blade that produces larger torques. This explains why the overall torque difference at $7 \mathrm{~m} / \mathrm{s}$ highlighted in Tab. II is smaller than at $13 \mathrm{~m} / \mathrm{s}$. Also, both coarse and fine grid analyses fail to predict the separation close to the hub, since both CFD analyses overestimate $C_{T}$ at the root.

Figures 5 and 6 compare computed and measured $c_{p}$ profiles at $\left|\underline{\mathrm{u}}_{\infty}^{A}\right|=7 \mathrm{~m} / \mathrm{s}$ and $\left|\underline{\mathrm{u}}_{\infty}^{A}\right|=13 \mathrm{~m} / \mathrm{s}$ respectively considering the radial positions $r / R=0.3,0.47,0.63,0.8$ and 0.95 . At $7 \mathrm{~m} / \mathrm{s}$ an excellent agreement is observed between both CFD simulations and experimental data, indicating that the coarse grid solution is fairly grid-independent for 

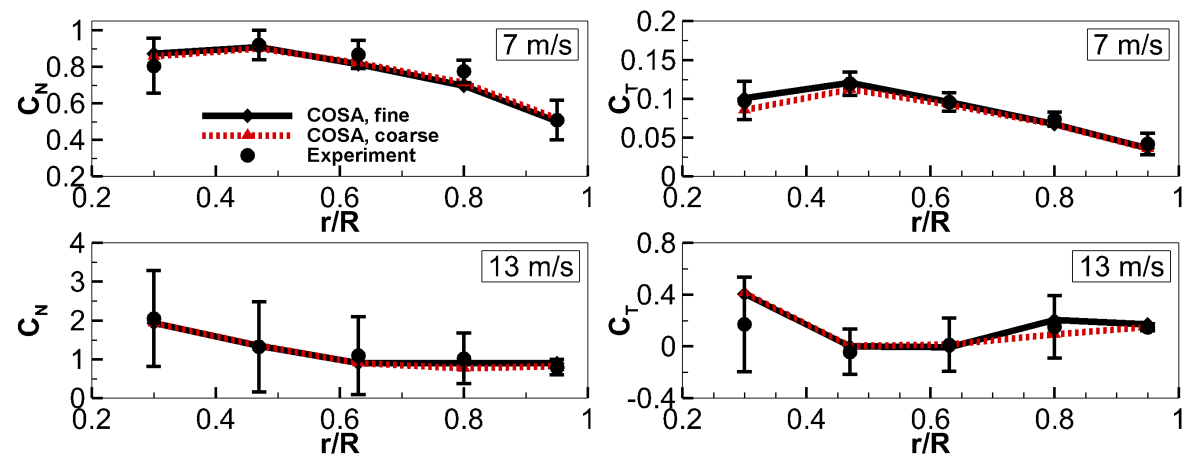

Figure 4. NREL Phase VI HAWT: measured and steady CFD spanwise profiles of the normal force coefficient $C_{N}$ (left) and tangential force coefficient $C_{T}$ (right) for $\left|\underline{u}_{\infty}^{A}\right|=7 \mathrm{~m} / \mathrm{s}$ and $\left|\underline{u}_{\infty}^{A}\right|=7 \mathrm{~m} / \mathrm{s}$ with zero yaw misalignment.

this operating condition. At $13 \mathrm{~m} / \mathrm{s}$, the agreement between the two CFD simulations and the experimental data is still fairly good, but worse than at $7 \mathrm{~m} / \mathrm{s}$, presumably due to higher aerodynamic loading. At $r / R=0.3$, both CFD simulations fail to predict the occurrence of stall, highlighted by the static pressure plateau on the suction side for $0<x^{\prime} / c<0.2$. Significant differences between the coarse and fine grid solutions exist at $r / R=0.8$, indicating that the coarse grid solution is not grid-independent for this operating condition. It is also noted that the fine grid $c_{p}$ profile is much closer to the measured data, whereas the coarse grid solution is flatter, indicating incorrectly a significant level of stall also at this large radius.
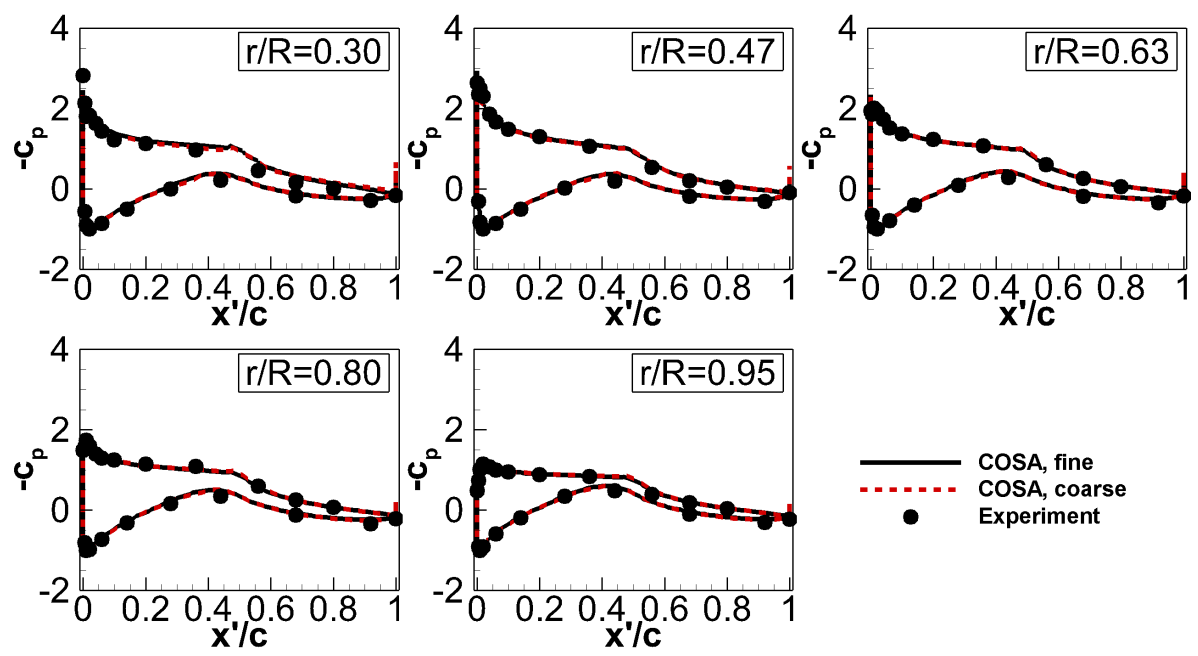

Figure 5. NREL Phase VI HAWT: measured and steady CFD blade pressure coefficient $c_{p}$ at five radial positions for $\left|\underline{u}_{\infty}^{A}\right|=7 \mathrm{~m} / \mathrm{s}$ and zero yaw misalignment.

Before discussing the considered yawed flow condition, some key parameters are introduced making use of the left and right schematics of Fig. 7, depicting respectively the top and front views of a HAWT in yawed wind. The blade azimuthal position is indicated by the angle $\theta=\Omega t$, measured from the vertical and descending point (position $A$ ), and the yaw misalignment is indicated by the angle $\delta$. With reference to nomenclature used in rotorcraft aerodynamics, the arc $\widehat{D A B}$ corresponds to the retreating leg and the arc $\widehat{B C D}$ corresponds to the advancing leg of the blade trajectory. The yawed condition examined below is that with $\left|\underline{u}_{\infty}^{A}\right|=7 \mathrm{~m} / \mathrm{s}$ and $\delta=30^{\circ}$, and was analyzed with $T D$ simulations using both the $4.2 \mathrm{M}$-cell and the $34 \mathrm{M}$-cell full rotor grids. A time-step sensitivity analysis showed that a time-step-independent solution could be obtained using 360 time-intervals per period. The number of revolutions required to achieve a fully periodic solution was 7 using a freestream initialization. Thrust and torque were used to monitor the periodicity error. The 

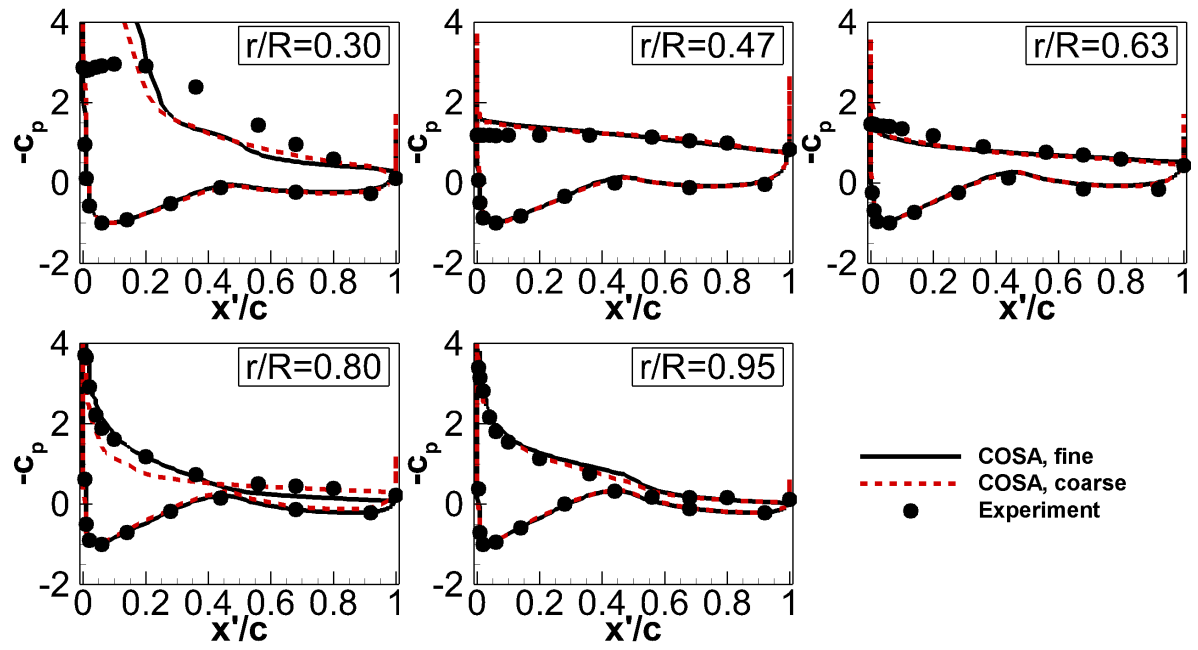

Figure 6. NREL Phase VI HAWT: measured and steady CFD blade pressure coefficient $c_{p}$ at five radial positions for $\left|\underline{\mathrm{u}}_{\infty}^{A}\right|=13 \mathrm{~m} / \mathrm{s}$ and zero yaw misalignment.

solution was taken to be periodic once, for both outputs, the maximum difference between the instantaneous values of the last two periods was less than $0.1 \%$ of the maximum value of the last period.
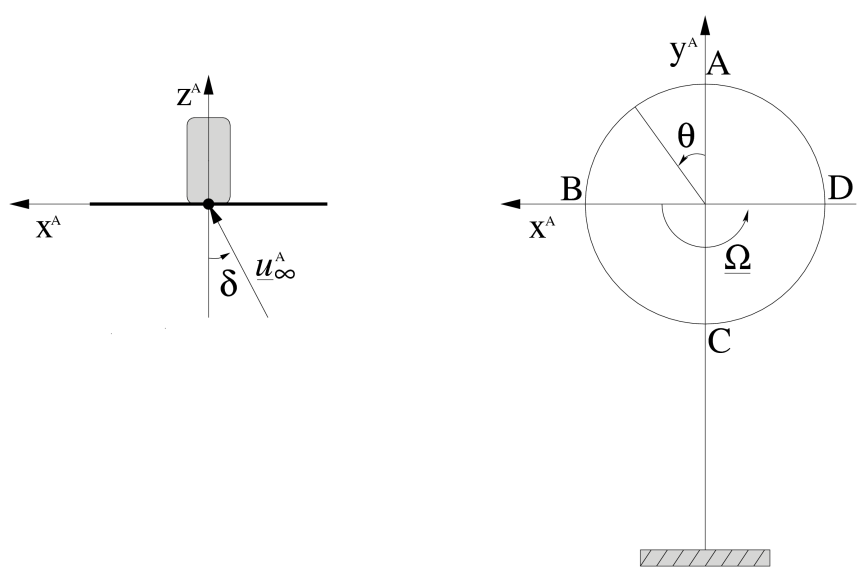

Figure 7. Schematic views of the HAWT in yawed wind operating regime. Left plot: top view; Right plot: front view.

Figure 8 compares the measured spanwise profiles of $C_{N}$ and $C_{T}$ at the azimuthal positions $\theta=210^{\circ}, \theta=270^{\circ}$ and $\theta=330^{\circ}$ with the $4.2 \mathrm{M}$-cell and the $34 \mathrm{M}$-cell grid TD CFD estimates. Simulations and measurements are in excellent agreement at all three azimuthal positions. The largest discrepancy is that of $C_{N}$ close to the blade root at $\theta=330^{\circ}$, but the difference remains within the bounds of the indicated experimental uncertainty. The coarse and fine grid results are in very good agreement, and some small differences only occur towards the root. Both CFD and measurements indicate that both the tangential and the normal forces on the blade sections increase from $\theta=210^{\circ}$ to $\theta=330^{\circ}$. This is due to the increment of the effective angle of attack (AoA) in this $\theta$ interval, as also obtainable with the simplified model of yawed HAWT aerodynamics in [24]. It is also seen that the tangential force varies substantially more with $\theta$ than the normal force does. The choice of the three angular positions above was made because the experimental measurements in yawed wind were affected by the wake of the instrumentation boom and enclosures [6] and the static pressure field of the tower [36] at other azimuthal positions.

Figure 9 compares computed and measured $c_{p}$ profiles at the radial positions $r / R=0.3,0.47$ and 0.8 for the azimuthal positions $\theta=210^{\circ}, \theta=270^{\circ}$ and $\theta=330^{\circ}$. Excellent agreement of simulations and measurements, and negligible 

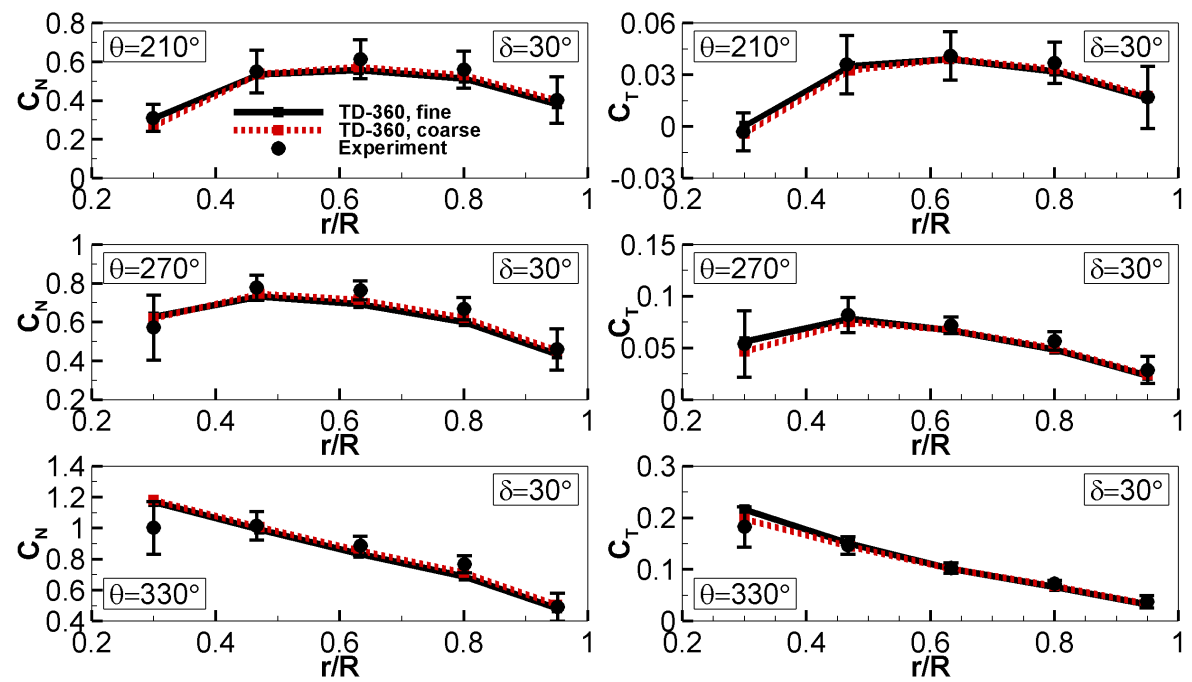

Figure 8. NREL Phase VI HAWT: measured and TD CFD spanwise profiles of normal force coefficient $C_{N}$ (left) and tangential force coefficient $C_{T}$ (right) at three azimuthal position for $\left|\underline{u}_{\infty}^{A}\right|=7 \mathrm{~m} / \mathrm{s} \mathrm{m} / \mathrm{s}$ and $\delta=30^{\circ}$.

differences between coarse and fine grid results are observed at all radial and circumferential positions. Both measurements and simulations highlight that the minimum blade loading among the considered azimuthal positions occurs at $\theta=210^{\circ}$, close to the midpoint of the advancing leg of the trajectory, and this loading is lower than at steady conditions, as noted by comparing these profiles with those of Fig. 5. At $r / R=0.3$ the particularly low value of the AoA indicated by the very
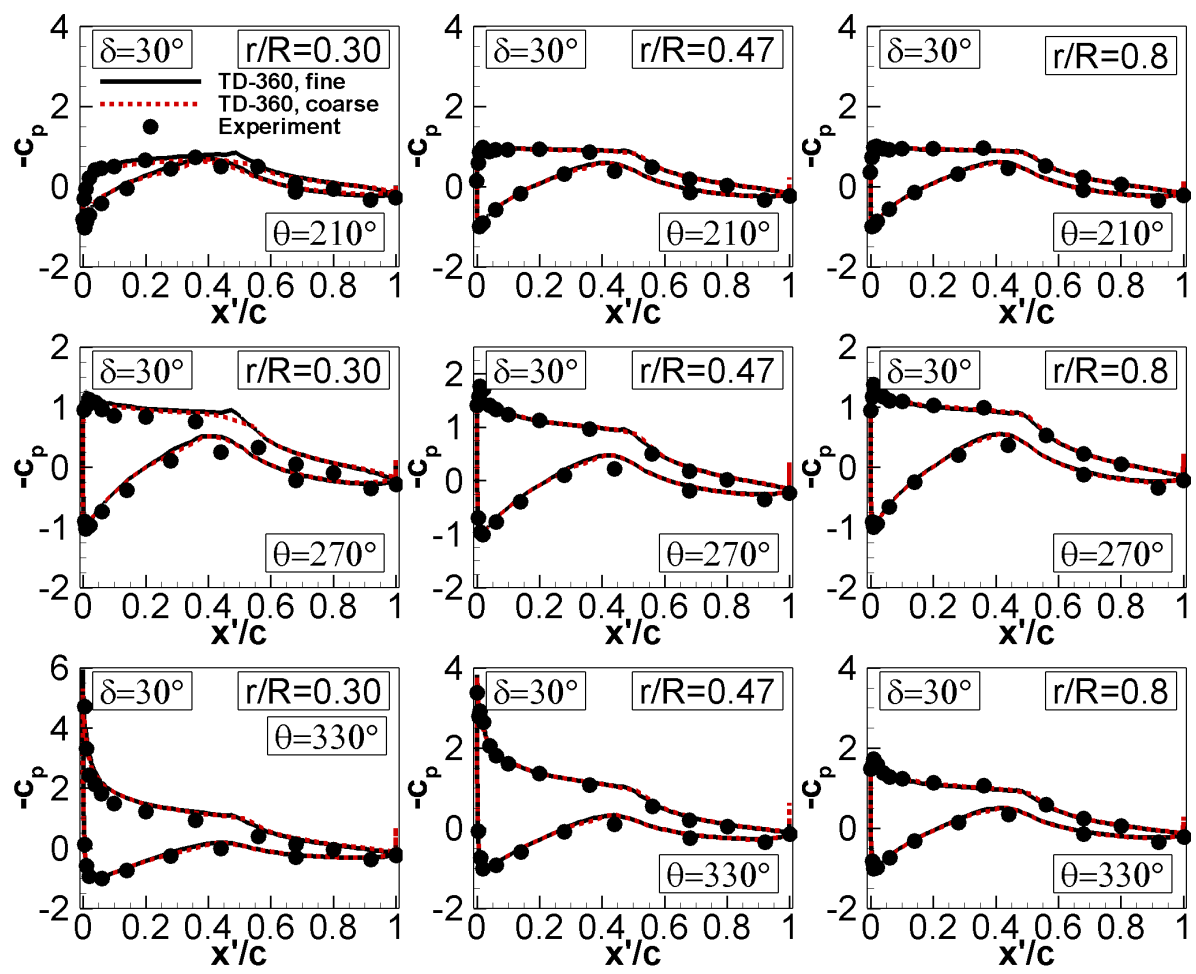

Figure 9. NREL Phase VI HAWT: measured and TD CFD blade pressure coefficient $c_{p}$ at three azimuthal and three radial positions for $\left|\underline{u}_{\infty}^{A}\right|=7 \mathrm{~m} / \mathrm{s}$ and $\delta=30^{\circ}$. 
small loading points to possible interactions of the blade with the rotor wake. The loading is instead maximum at $\theta=330^{\circ}$, close to the midpoint of the retreating leg of the trajectory, due to AoA along the blade achieving its maximum [20].

\section{RESULTS}

\subsection{NREL Phase VI turbine}

Here the yawed wind condition of the NREL Phase VI HAWT analyzed above with $T D$ simulations using 360 timeintervals per period is analyzed with the $H B$ solver using a number of complex harmonics $N_{H}$ from 1 to 4 . Making use of the MFPBC described in subsection 4.3, a single $180^{\circ}$ was used for all $H B$ analyses. Given the very good agreement between coarse and fine grid $T D$ analyses, the coarse grid is used herein.

The periodic profiles of the blade thrust coefficient $C_{F_{z}}$ and the blade torque coefficient $C_{M_{z}}$ computed by the $T D-360$ and the four $H B$ analyses are reported in the left and right plots of Fig. 10 respectively. The definitions of these coefficients are:

$$
\begin{aligned}
C_{F_{z}} & =\frac{F_{z}}{0.5 \rho_{\infty}\left|\underline{\mathrm{u}}_{\infty}^{A}\right|^{2} \pi R^{2}} \\
C_{M_{z}} & =\frac{M_{z}}{0.5 \rho_{\infty}\left|\underline{\mathrm{u}}_{\infty}^{A}\right|^{2} \pi R^{3}}
\end{aligned}
$$

It is seen that the $H B-3$ profiles differ negligibly from the $T D-360$ profiles, indicating that 3 complex harmonics are sufficient to capture the main dynamics of this regime. It is also observed that the $C_{F_{z}}$ profile is bimodal, presenting a local minimum at $\theta \approx 180^{\circ}$. As highlighted by the $c_{p}$ profiles at $\theta=210^{\circ}$ (Fig. 9), this is due to very low AoA along the blade and small interactions of the blade with the rotor wake.
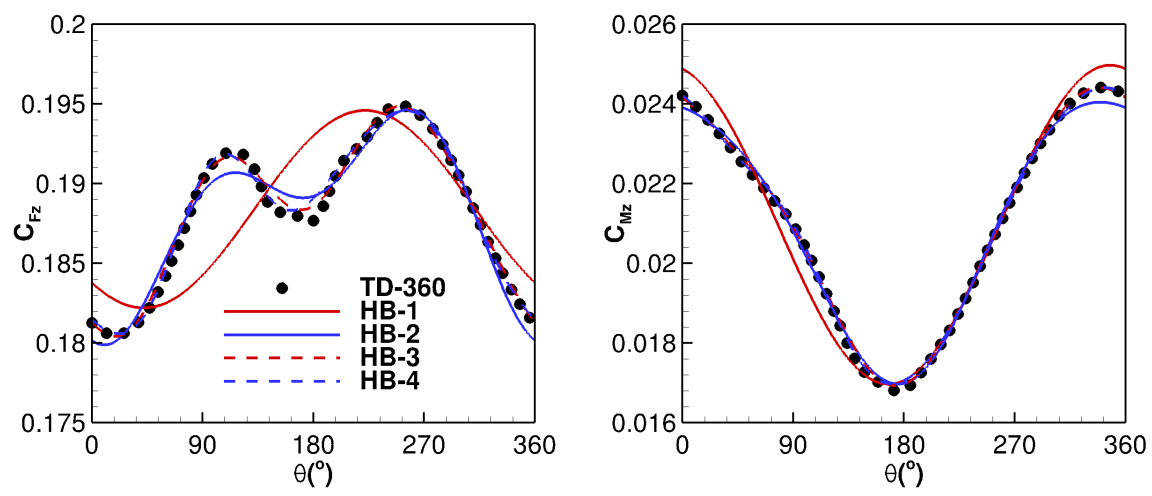

Figure 10. NREL Phase VI: the time dependent thrust $C_{F_{z}}$ and torque $C_{M_{z}}$ coefficients.

The left and right plots of Fig. 11 present respectively the profiles of $C_{N}$ and $C_{T}$ along the blade for $\theta=0^{\circ}, \theta=110^{\circ}$ and $\theta=180^{\circ}$. These results confirm that 3 complex harmonics are sufficient to achieve a frequency-domain solution differing negligibly from the $T D$ solution, since all $H B-3$ and $T D-360$ profiles are nearly superimposed. It is also seen that the trends highlighted in Fig. 11 are similar to those of Fig. 8, in that both the tangential and the normal force decrease with qualitatively similar patterns from the highest to the lowest vertical positions along both trajectory legs $\widehat{A B C}$ and $\widehat{C D A}$.

The $c_{p}$ profiles at $r / R=0.3,0.47$ and 0.8 for the azimuthal positions considered above are reported in Fig. 12. These plots show that the $H B$ solver resolves properly the blade static pressure for most radial and azimuthal positions using just 1 or 2 complex harmonics. At $\theta=180^{\circ}$ and $r / R=0.3$, however, only the $H B-3$ simulation predicts correctly the 

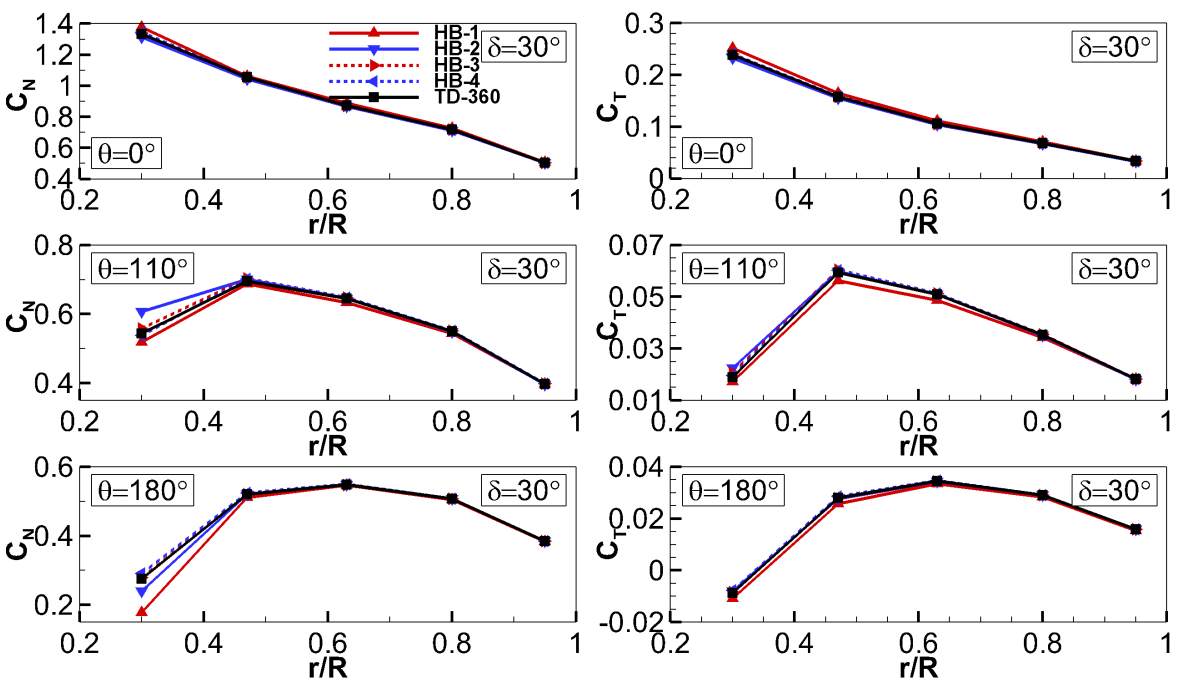

Figure 11. NREL Phase VI HAWT: $T D$ and $H B$ spanwise profiles of normal force coefficient $C_{N}$ (left) and tangential force coefficient $C_{T}$ (right) at three azimuthal position for $\left|\underline{u}_{\infty}^{A}\right|=7 \mathrm{~m} / \mathrm{s} \mathrm{m} / \mathrm{s}$ and $\delta=30^{\circ}$.

blade static pressure, and large differences among the $H B-1, H B-2$ and $H B-3$ predictions exist. This is due to the more complex dynamics at this position due to very low effective AoA and local interactions of the blade with the rotor wake.
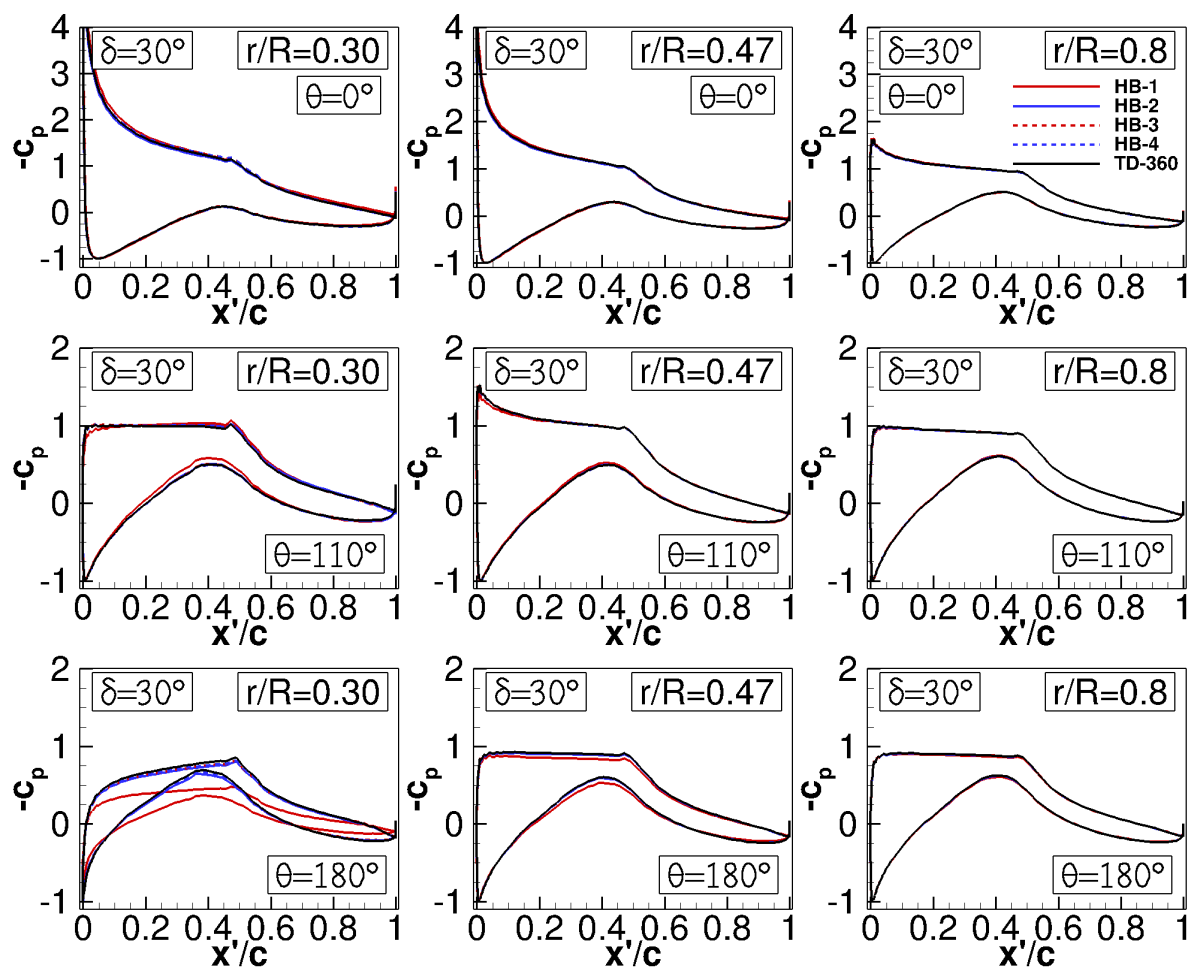

Figure 12. NREL Phase VI HAWT: $T D$ and $H B$ CFD blade pressure coefficient $c_{p}$ at three azimuthal and three radial positions for $\left|\underline{\mathrm{u}}_{\infty}^{A}\right|=7 \mathrm{~m} / \mathrm{s}$ and $\delta=30^{\circ}$. 
Table III. NREL Phase VI HAWT: $H B$ speed-up.

\begin{tabular}{|c|c|c|c|c|c|c|}
\hline & HB 1 & HB 2 & HB 3 & HB 4 & TD-360 & steady \\
\hline speed-up & 74.7 & 43.6 & 31.0 & 24.0 & 1.0 & - \\
\hline
\end{tabular}

Table III reports the $H B$ speed-up parameter, defined as the ratio of the runtime of the $T D-360$ simulation and the $H B$ analysis with $N_{H}$ harmonics, and shows that the $H B 3$ simulation, which is in excellent agreement with the $T D-360$ simulation, reduces the analysis runtime by a factor 31 .

\subsection{NREL 5 MW turbine}

Here a yawed wind condition of the 3 -blade 126 m-diameter NREL $5 \mathrm{MW}$ wind turbine is considered. The selected operating point assumes $\delta=20^{\circ},\left|\underline{u}_{\infty}^{a}\right|=11.4 \mathrm{~m} / \mathrm{s}$ and $\Omega=12.1 \mathrm{RPM}$, giving a tip-speed ratio of 7 . The CFD model includes only the rotor with zero cone angle and zero vertical shear of the wind. The Reynolds number based on the standard density of $1.225 \mathrm{~kg} / \mathrm{m}^{3}$, the tip chord, and the relative tip velocity is $6.5 \times 10^{6}$. Yawed wind simulations were performed using the $T D$ and $H B$ solvers.

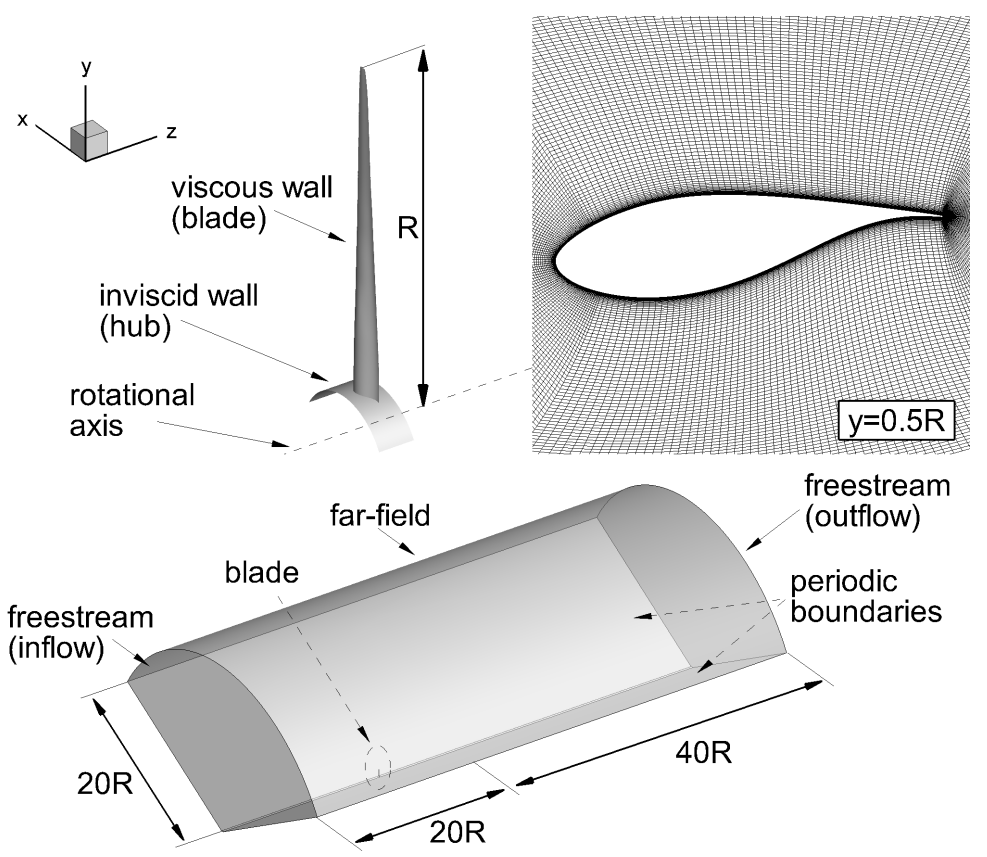

Figure 13. NREL 5 MW HAWT: blade geometry (top left), airfoil grid at $50 \%$ of tip radius (top right), and domain dimensions and boundary conditions (bottom).

Views of the blade geometry and the sector grid used for the $H B$ analyses discussed below are reported in Fig. 13. A fine 21, 585, 920-cell multi-block sector grid was generated first. The mesh past each airfoil has an O-grid topology, featuring 256 cells on the airfoil, 112 cells in the normal-like direction, and $d_{w}=2 \cdot 10^{-6} c$. The grid has 128 cells along the blade length and 128 cells from the tip to the cylindrical far field. On the blade surface, the grid is clustered in the spanwise direction towards both the root and the tip. The blade tip has a sharp-tip topology. Fig. 13 also reports the dimensions of the physical domain and the BCs applied to its boundaries. The distance of the far field boundaries from the rotor center were selected following the guidelines of [37]. The top left plot of Fig. 13 shows that also in this case the hub was modeled as a zero-thickness axially-short cylindrical surface, and an inviscid wall BC was used on its inner and outer sides. 
A coarse 2.7 M-cell sector grid was obtained from the fine sector grid. Negligible differences were observed in steady flow analysis of this rotor with zero yaw misalignment, and therefore the coarse grid was used for all analyses reported below. The $H B$ simulations were performed using the sector grid with MFPBC enforced on the two periodic boundaries, whereas the $T D$ analyses used a full rotor $8.1 \mathrm{M}$-cell grid obtained by creating two additional rotor sectors. A timestep refinement showed that 360 steps per period were sufficient to determine a fully periodic time-step-independent solution for $\delta=20^{\circ}$. The periodicity error of the simulation was monitored using the rotor thrust and torque as explained in Section 5, and 12 revolutions were required to fulfill the $0.1 \%$ periodicity error constraint of both output. Further information on the mesh generation and refinement analyses for this test case are available in [35].
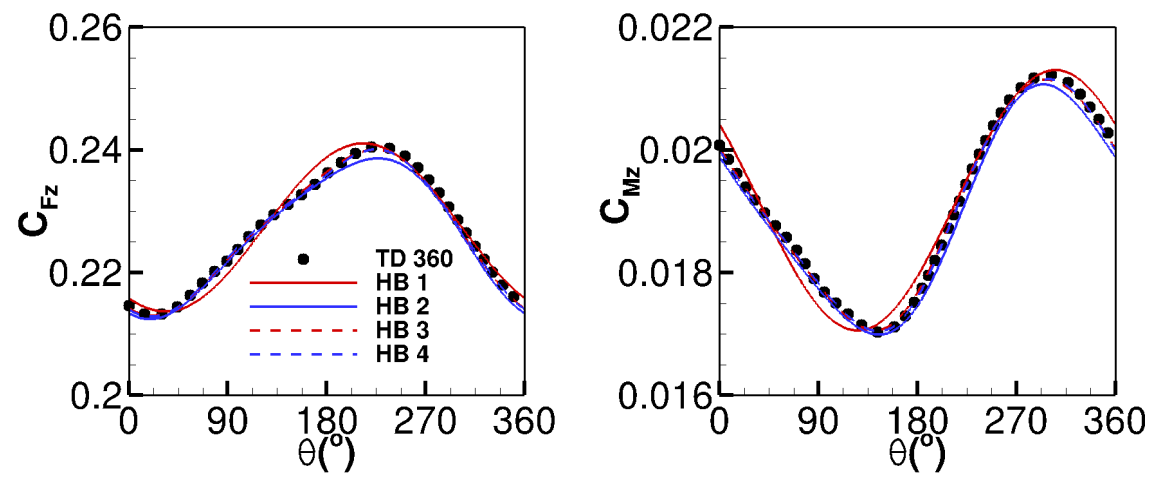

Figure 14. NREL 5MW: the thrust $C_{F_{z}}$ and the torque $C_{M_{z}}$ coefficients.

Figure 14 compares the evolution over one revolution of the thrust coefficient $C_{F_{z}}$ and the torque coefficient $C_{M_{z}}$ computed with the $T D-360$ and the $H B$ simulations using between 1 and 4 harmonics. One notes that the profiles of both variables determined with the $T D$ and the $H B-3$ analyses are indistinguishable, indicating that the yawed flow dynamics of this regime is fully resolved using just 3 harmonics. One also notices cyclic variations of the rotor thrust of about $7 \%$ and cyclic variations of the torque of about $10 \%$, which yield stress cycles which may reduce the fatigue life of HAWT blades and drivetrain.

The spanwise distributions of the $C_{N}$ and $C_{T}$ at the azimuthal positions $\theta=0^{\circ}, \theta=90^{\circ}$ and $\theta=180^{\circ}$ computed by the $T D$ and four $H B$ analyses are compared in Fig. 15 These plots show that, for this regime, one harmonic captures fairly well the blade loads over most part of its length and that a larger number of harmonics is required to improve the $H B$ prediction accuracy also in the root region, where more flow nonlinearities occur due to lower Reynolds numbers and high AoA. These small nonlinearities also account for the differences among the four $H B$ predictions in Fig. 14.

Table IV. NREL 5 MW HAWT: $H B$ speed-up.

\begin{tabular}{|c|c|c|c|c|c|c|}
\hline & HB 1 & HB 2 & HB 3 & HB 4 & TD-360 & steady \\
\hline speed-up & 115.3 & 67.2 & 47.8 & 37.0 & 1.0 & - \\
\hline
\end{tabular}

Table IV shows that the speed-up of the $H B$ simulations, and highlights that the $H B-3$ analysis, which has been shown to feature an accuracy comparable with that of the $T D$ analysis, is about 50 times faster than the latter method.

\section{CONCLUSIONS}

A novel harmonic balance Navier-Stokes approach to the analysis of wind turbine 3D turbulent periodic flows using the shear stress transport turbulence model has been presented, with particular emphasis on modeling aspects relevant to the 

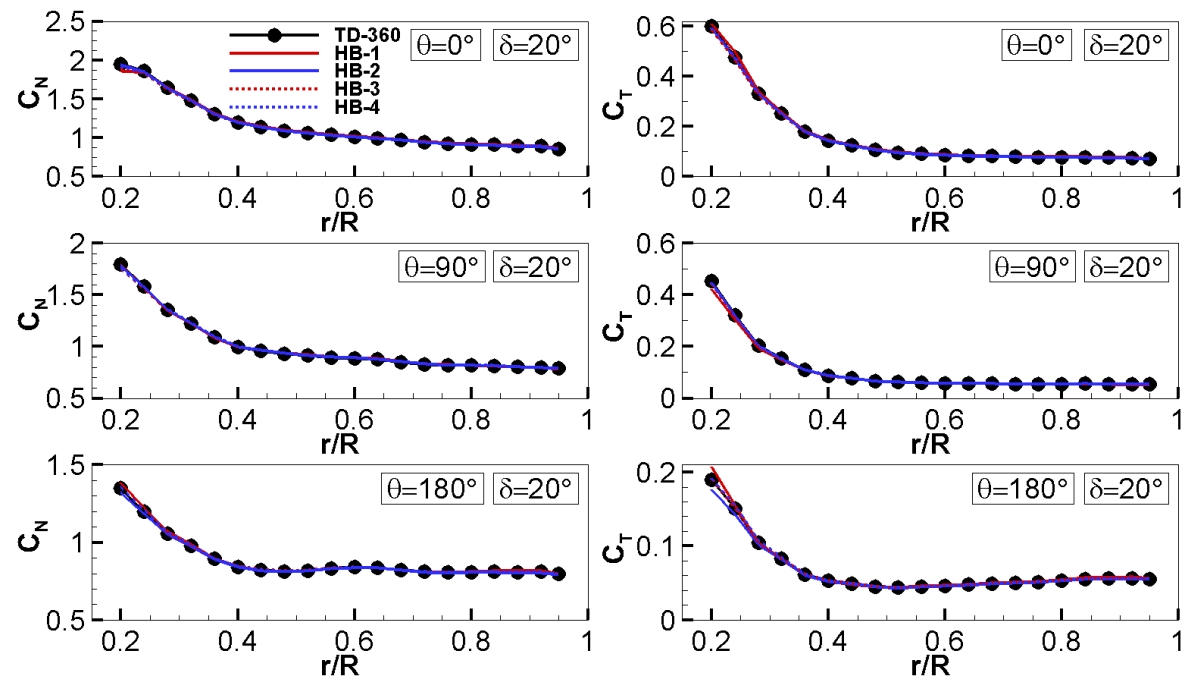

Figure 15. NREL 5 MW HAWT: $T D$ and $H B$ spanwise profiles of normal force coefficient $C_{N}$ (left) and tangential force coefficient $C_{T}$ (right) at three azimuthal position for $\left|\underline{u}_{\infty}^{A}\right|=11.4 \mathrm{~m} / \mathrm{s} \mathrm{m} / \mathrm{s}$ and $\delta=20^{\circ}$.

implementation of this technology in other CFD codes. The predictive capabilities of the underlying steady and timedomain codes have been validated using respectively steady and yawed wind regimes of the NREL Phase VI turbine experiment. The computational efficiency of the new 3D $H B$ solver has been assessed by computing yawed wind flows of the NREL Phase VI and the NREL 5 MW turbines, and it has been shown that the $H B$ NS CFD method can reduce the runtime of periodic flow simulations by up to 50 times over the standard $T D$ method. This acceleration occurs because $H B$ method does not require the solution of lengthy transients occurring in $T D$ simulations before achieving the sought periodic state, and can use reduced-size analysis domains (sector grids) making use of an ad-hoc multifrequency periodicity boundary condition. This achievement is believed to bring the use of NS CFD closer to the industrial development of multi-megawatt wind turbines, particularly to support the analysis of several types of fatigue-inducing loads, and also rotor aeroelastic analyis and design.

The analysis of strongly non-periodic flow regimes, like those associated with high levels of atmospheric turbulence, will continue to require the conventional time-domain approach, and this may delay the industrial deployment of highfidelity CFD for these particular problems. It should be noted, however, that the $H B$ CFD technology has the potential of analysing efficiently wind turbine flows more general than those considered in this study, namely problems characterized by an excitation featuring several co-prime fundamental frequencies. These extensions have been proposed initially for turbomachinery problems, and one of the available methods is reported in [38], which also reviews other methods for solving these problems.

\section{ACKNOWLEDGEMENT}

The authors thank EPSRC for providing access to computational resources on ARCHER through the UK Applied Aerodynamics Consortium Leadership Project e529. Use was also made of the facilities of N8 HPC Center of Excellence funded by EPSRC Grant No.EP/K000225/1. 


\section{REFERENCES}

1. Sørensen N, Johansen J. UPWIND: aerodynamics and aero-elasticity rotor aerodynamics in atmospheric shear flow. Proceedings of The European Wind Energy Conference \& Exhibition, EWEC 2007 May 2007. European Wind Energy Conference \& Exhibition, EWEC 2007.

2. Manwell J, McGowan J, Rogers A. Wind Energy Explained. Theory, Design and Application. John Wiley and Sons Ltd., 2002.

3. Bak C. Aerodynamic design of wind turbine rotors. Advances in wind turbine blade design and materials, Energy, vol. 47, Gentzsch W, Harms U (eds.). Woodhead Publishing: Cambridge, UK, 2013; 59-108.

4. Himmelskamp H. Profile investigations of a rotating airscrew. PhD Dissertation Goettingen 1945, Report and Translation 832. MAP Voelkenrode, 1947.

5. Simms DA, Schreck S, Hand L, Fingersh LJ. Nrel Unsteady Aerodynamics Experiment in the NASA-Ames Wind tunnel: A Comparison of Predictions to Measurements. Technical Report NREL/TP-500-29494, NREL, Golden, CO, USA June 2001.

6. Hand MM, Simms DA, Fingersh LJ, Jager DW, Cotrell JR, Schreck S, Larwood SM. Unsteady aerodynamics experiment phase vi: Wind tunnel test configurations and available data campaigns. Technical Report NREL/TP500-29955, NREL, Golden, CO, USA Dec 2001.

7. Snel H, Schepers JG, Montgomerie B. The mexico project (model experiments in controlled conditions): The database and first results of data processing and interpretation. Journal of Physics: Conference Series 2007; 75(1):012 014.

8. Madsen H, Sørensen N, Schreck S. Yaw Aerodynamics Analyzed with Three Codes in Comparison with Experiments. AIAA paper 2003-0519 January 2003. 41st AIAA Aerospace Sciences Meeting and Exhibit, Reno, Nevada.

9. Le Pape A, Gleize V. Improved Navier-Stokes Computations of a Stall-regulated Wind Turbine Using Low Mach Number Preconditioning. AIAA paper 2006-1502 January 2006. 44th AIAA Aerospace Sciences Meeting and Exhibit, Reno, Nevada.

10. Sezer-Uzol N, Gupta A, Long L. 3D Time-Accurate Inviscid and Viscous cfd Simulations of Wind Turbine Rotor Flow Fields. Parallel Computational Fluid Dynamics 2007, Lecture Notes in Computational Science and Engineering, vol. 67, Tuncer I, Guelcat U, Emerson D, (Eds) KM (eds.), Springer-Verlag, 2007; 457-464.

11. Tsalicoglou C, Jafari S, Chokani S, Abhari R. Rans computations of wind turbine near-wake aerodynamics in uniform and yawed inflow. ASME paper GT2013-96034 June 2013.

12. Yu D, You J, Kwon O. Numerical investigation of unsteady aerodynamics of a horizontal-axis wind turbine under yawed flow conditions. Wind Energy 2013; 16(5):711-727.

13. Schepers J, Boorsma K, Gomez-Iradi S, Schaffarczyk P, Madsen H, Sørensen N, Shen W, Lutz T, Schulz C, Herraez I, et al.. Final report of IEA wind task 29: Mexnext (phase 2). Technical Report ECN-E-14-060, ECN, Petten, Netherlands 2014.

14. Hall K, Thomas J, Clark W. Computations of Unsteady Nonlinear Flows in Cascades Using a Harmonic Balance Technique. AIAA Journal May 2002; 40(5):879-886.

15. van der Weide E, Gopinath A, Jameson A. Turbomachinery Applications with the Time Spectral Method. AIAA paper 2005-4905 June 2005. 17th AIAA Computational Fluid Dynamics Conference, Torontp, Ontario, Canada.

16. Su X, Yuan X. Implicit Solution of Time-Spectral Method for Periodic Unsteady Flows. International Journal for Numerical Methods in Fluids 2010; 63(7):860-876.

17. Da Ronch A, McCracken A, Badcock K, , Widhalm M, Campobasso M. Linear Frequency Domain and Harmonic Balance Predictions of Dynamic Derivatives. Journal of Aircraft 2013; 50(3):694-707.

18. Sicot F, Puigt G, Montagnac M. Block-Jacobi Implicit Algorithms for the Time Spectral Method. AIAA Journal December 2008; 46(12):3080-3089.

19. Woodgate MA, Badcock KJ. Implicit Harmonic Balance Solver for Transonic Flows with Forced Motions. AIAA Journal April 2009; 47(4):893-901. 
20. Campobasso M, Baba-Ahmadi M. Analysis of Unsteady Flows Past Horizontal Axis Wind Turbine Airfoils Based on Harmonic Balance Compressible Navier-Stokes Equations with Low-Speed Preconditioning. Journal of Turbomachinery November 2012; 134(6).

21. Howison J, Ekici K. Unsteady Analysis of Wind Turbine Flows Using the Harmonic Balance Method. AIAA paper 2013-1107 January 2013. 51st AIAA Aerospace Sciences Meeting including the New Horizons Forum and Aerospace Exposition, Grapewine (Dallas/Ft. Worth Region), Texas.

22. Howison J. Aeroelastic Analysis of a Wind Turbine Blade Using the Harmonic Balance Method. PhD Thesis, University of Tennessee, Knoxville, United States December 2015.

23. Howison J, Ekici K. Dynamic stall analysis using harmonic balance and correlation based $\gamma-r e_{\theta}$ transition models for wind turbine applications. Wind Energy 2015; 18:2047-2063.

24. Campobasso M, Gigante F, Drofelnik J. Turbulent Unsteady Flow Analysis of Horizontal Axis Wind Turbine Airfoil Aerodynamics Based on the Harmonic Balance Reynolds-Averaged Navier-Stokes Equations. ASME paper GT201425559 June 2014.

25. Jonkman J, Butterfield S, Musial W, Scott G. Definition of a 5-MW Reference Wind Turbine for Offshore System Development. Technical Report NREL/TP-500-38060, NREL, Golden, CO, USA 2009.

26. Campobasso M, Drofelnik J, Gigante F. Comparative assessment of the harmonic balance Navier-Stokes technology for horizontal and vertical axis wind turbine aerodynamics. Computers \& Fluids 2016; 136:354 - 370.

27. Chen J, Ghosh A, Sreenivas K, Whitfield D. Comparison of computations using navier-stokes equations in rotating and fixed coordinates for flow through turbomachinery. 35th Aerospace Sciences Meeting and Exhibit January 1997. Reno, NV, U.S.A.

28. Campobasso M, Piskopakis A, Drofelnik J, Jackson A. Turbulent Navier-Stokes Analysis of an Oscillating Wing in a Power-Extraction Regime Using the Shear Stress Transport Turbulence Model. Computers and Fluids December 2013; 88:136-155.

29. Drofelnik J, Campobasso M. Comparative turbulent three-dimensional navier-stokes hydrodynamic analysis and performance assessment of oscillating wings for renewable energy applications. International Journal of Marine Energy 2016; 16:100 - 115.

30. Balduzzi F, Drofelnik J, Bianchini A, Ferrara G, Ferrari L, Campobasso M. Darrieus wind turbine blade unsteady aerodynamics: a three-dimensional navier-stokes cfd assessment. Energy 2017; 128:550-563.

31. Obayashi S. Freestream capturing for moving coordinates in three dimensions. AIAA Journal 1992; 30 (4):1125 1128.

32. Menter F. Two-equation Turbulence-models for Engineering Applications. AIAA Journal August 1994; 32(8):15981605.

33. Jameson A, Baker T. Solution of the Euler Equations for Complex Configurations. AIAA paper 83-1929 July 1983. 6th AIAA Computational Fluid Dynamics Conference, Danvers, Massachusetts.

34. Ekici K, Hall KC, Dowell EH. Computationally fast harmonic balance methods for unsteady aerodynamic predictions of helicopter rotors. Journal of Computational Physics 2008; 227(12):6206 - 6225.

35. Drofelnik J. Massively parallel time- and frequency-domain navier-stokes computational fluid dynamics analysis of wind turbine and oscillating wing unsteady flows. PhD Thesis, University of Glasgow, United Kingdom 2017.

36. Gomez-Iradi S. CFD for Horizontal Axis Wind Turbines. PhD Thesis, University of Liverpool, United Kingdom 2009.

37. Chow R, van Dam C. Verification of computational simulations of the nrel $5 \mathrm{mw}$ rotor with a focus on inboard flow separation. Wind Energy 2012; 15(8):967-981.

38. Guedeney T, Gomar A, Gallard F, Sicot F, Dufour G, Puigt G. Non-uniform time sampling for multiple-frequency harmonic balance computations. Journal of Computational Physics 2013; 236:317-345. 